\title{
Identification of weak buses using Voltage Stability Indicator and its voltage profile improvement by using DSTATCOM in radial distribution systems
}

\author{
S M Suhail Hussain ${ }^{1}, \mathrm{~N}_{\text {Visali }}^{2}$ \\ ${ }^{1,2}$ (Department of Electrical and Electronics, JNTUA college of Engineering, Pulivendula INDIA)
}

\begin{abstract}
This paper presents a method to determine the weakest bus in a distribution network. The voltage stability indicator (VSI) identifies the weakest bus of system. The voltage stability indicator is derived from the voltage equation of radial distribution system. The weakest bus voltage profile is improved by placing a DSTATCOM. The DSTATCOM is modeled to supply the required reactive power for compensation and to maintain the voltage magnitude of the node where DSTATCOM is placed as 1 p.u. The validity of the proposed VSI and DSTATCOM modeling is examined by a standard 33 bus radial distribution system. The results validate the proposed VSI and DSTATCOM models in large distribution systems.
\end{abstract}

Keywords - Distribution system, DSTATCOM, load flow, voltage stability, voltage stability indicator (VSI).

\section{INTRODUCTION}

Voltage stability is an important factor to be considered in power system operation and planning since voltage instability would lead to system collapse. The problem of voltage stability [1] has been defined as inability of the power system to provide the reactive power [2] or non-uniform consumption of reactive power by the system itself. Therefore, voltage stability is a major concern in planning and assessment of security of large power systems in contingency situation, especially in developing countries because of non-uniform growth of load demand and lacuna in the reactive power management side [3]. The loads generally play a key role in voltage stability analysis and therefore the voltage stability is known as load stability.

Radial distribution systems have high resistance to reactance ratio, which causes high power loss. Hence, the radial distribution system is one of the power systems, which may suffer from voltage instability. In this paper a technique for determination of voltage stability at load bus is presented. Using this Voltage Stability indicator, the buses of the system which are weak in nature can be identified. So far much attention was paid on voltage stability analysis of transmission lines, researchers have paid very little attention to develop a voltage stability indicator for radial distribution systems $[4,5,6]$.

In radial distribution system, providing demanding power to the entire load while maintaining voltage magnitude at an acceptable range is one of the major system constraints. There are two principal conventional means of maintaining voltages at an acceptable range in distribution systems are series voltage regulators and shunt capacitors. Conventional series voltage regulators are commonly used for voltage regulation in distribution system $[7,8,9]$. But these devices cannot generate reactive power and by its operation only force the source to generate requested reactive power and they have quite slow response as these operations are step by step [10]. Shunt capacitors can supply reactive power to the system but they are not capable to generate continuously variable reactive power. Another difficulty associated with the application of distribution capacitors is the natural oscillatory behavior of capacitors when they are used in the same circuit with inductive components. Which results in the well-known phenomena of ferroresonance and/or self-excitation of induction machinery [10].

The concept of FACTS devices was originally developed for transmission systems, but similar idea has been started to be applied to distribution systems. Distribution STATCOM (D-STATCOM) is a shunt connected voltage source converter which has been utilized to compensate power quality problems such as unbalanced load, voltage sag, voltage fluctuation and voltage unbalance. D-STATCOM is also utilized for the improvement of another aspect of power quality, i.e. voltage compensation in long term.

Section 2 presents the derivation of Voltage stability indicator, load flow of distribution system and mathematical modeling of DSTATCOM. In Section 3 the VSI and DSTATCOM are tested on a 33 bus radial distribution system and results are analysed. Section 4 summarizes the main points and results of the paper.

II.

BASIC THEORY

2.1 Derivation of voltage stability indicator

VSI is derived from voltage equation of the radial distribution system. The proposed indicator is given below considering a line of impedance $\mathrm{R}+\mathrm{j} \mathrm{X}$ is connected between two nodes as shown in the following Fig. 1 where $i$ and $j$ are respectively two nodes of the branch and node $i$ is sending end node [V(i)] and node $j$ is 
Identification of weak buses using Voltage Stability Indicator and its voltage profile improvement by receiving end node $[\mathrm{V}(\mathrm{j})]$ Therefore, power flow direction is from node $i$ to node $j$. The load at node $j$ is $\{\mathrm{P}(\mathrm{j})+\mathrm{jQ}(\mathrm{j})\}$. The impedance of the branch is $R(\mathrm{i})+\mathrm{jQ}(\mathrm{i})$ If line shunt admittances are neglected, the current flowing through the line is given by:

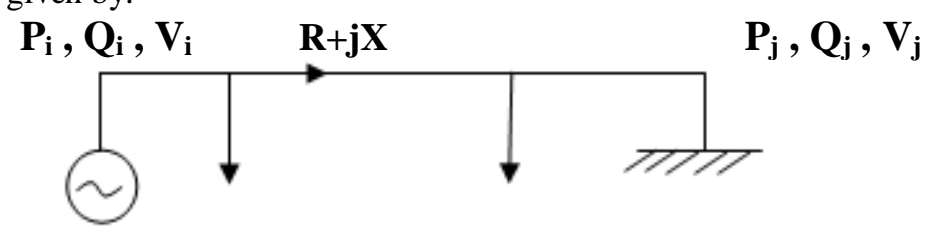

Figure 1: Radial system

$l(i)=\frac{V(i)<\theta_{i}-V(j)<\theta_{j}}{R(i)+j X(i)}$

The complex power is given as: $\mathrm{S}=\mathrm{VI} *$

$l(i)=\frac{P(j)-j Q(j)}{V^{*}(j)}$

Equating (1) and (2)

$\frac{V(i) \angle \theta_{i}-V(j)<\theta_{j}}{R(i)+j X(i)}=\frac{P(j)-j Q(j)}{V(j) \angle-\theta_{j}}$

$\left[V(i)<\theta_{i}-V(j)<\theta_{j}\right]\left[V(j)<-\theta_{j}\right]=[P(j)-j Q(j)][R(i)-j X(i)]$

Equating real and imaginary parts of (3), we get

$V(i) * V(j) \cos \left(\theta_{i}-\theta_{j}\right)-V(j)^{2}=P(j) * R(i)+Q(j) * X(i)$

$X(i) * P(j)-R(i) * Q(j)=V(i) * V(j) \sin \left(\theta_{i}-\theta_{j}\right)$

As in radial distribution systems voltage angles are negligible, hence $\left(\theta_{i}-\theta_{j}\right) \approx 0$, equation (4), (5) becomes

$V(i) * V(j)-V(j)^{2}=P(j) * R(i)+Q(j) * X(i)$

$X(i)=\frac{R(i) * Q(j)}{P(j)}$

From equation (6), (7)

$V(j)^{2}-V(i) * V(j)+P(j) R(i)+\frac{R(i) Q(j)^{2}}{P(j)}=0$

$V(j)^{2}-V(i) * V(j)+\frac{R(i)\left[P(j)^{2}+Q(j)^{2}\right]}{P(j)}=0$

The roots of equation (6) are real if

$V(i)^{2}-\frac{4 R(i)\left\{P(j)^{2}+Q(j)^{2}\right\}}{P(j)} \geq 0$

$\frac{4 R(i)\left\{P(j)^{2}+Q(j)^{2}\right\}}{V(i)^{2} P(j)} \leq 1$

$V S I=\frac{4 R(i)\left\{P(j)^{2}+Q(j)^{2}\right\}}{V(i)^{2} P(j)}$

Hence equation (11) is termed as voltage stability indicator (VSI). For stability of particular node the value of VSI must be VSI $\leq 1$. The range of VSI values is $0<\mathrm{VSI} \leq 1$. If the value of VSI approaches or greater than unity, then that node is highly unstable.

\subsection{Load flow technique:}


In this paper, backward forward sweep load flow technique is used to compute voltages and power flow for a radial distribution system. Several methods have been developed based on the concept of doing backward forward sweeps of radial distribution networks $[11,12,13]$.

Forward backward sweep based power flow algorithms generally take advantage of the radial network topology and consist of forward or backward sweep processes. In these algorithms, forward sweep is mainly the node voltage calculation from sending end to the far end of the feeder and laterals, and the backward sweep is primarily the branch current or power summation from far end to sending end of the feeder and laterals.

The first step to perform load flow is to create a matrix $M$ (with columns as nodes and rows as branches). For a particular branch (row) the sending end node of a branch is assigned -1 and receiving end as +1 , remaining elements as zero. From that M matrix the column which doesn't have -1 represents end node. After identifying all end nodes, the back propagation path from end node to source node must be identified. After finding end node corresponding row gives the branch attached to it and in this row, -1 value is identified. The corresponding column gives the sending end node to the studied branch. This searching process continues until the algorithm reaches a column which has no element equal to 1 . This represents source node. Hence the end nodes and back propagation paths for end nodes are calculated.

Most of the distribution system power flow algorithms employ KVL and KCL to calculate node voltages in forward and backward processes. The radial part is solved by a straight forward two-step procedure in which branch currents are first computed (backward sweep) and then bus voltages are updated (forward sweep).

Voltage at node $\mathrm{i}$ can be expressed as

$V(i)=V(i-1)-I(i) Z(i)$

The load current at node $i$ is given as

$I_{L}=\frac{P_{L}(i)-Q_{L}(i)}{V^{*}(i)}$

Branch current can be given as

$I(i)=I_{L}(i)+\sum_{j \in \beta_{i}} I(j)$

Algorithm for radial distribution system load flow:

1. Read the distribution system line data and load data

2. Form the node and branch matrix M.

3. Get the end nodes and the back propagation paths.

4. Obtain the value of $\beta$ of equation (14) by calculating the downstream nodes of every node.

5. Make a flat start by assuming the voltage profile of all buses to be 1 p.u.

6. Calculate load current $\mathrm{I}_{\mathrm{L}}(\mathrm{i})$ of each bus using equation (13)

7. Summation of all the load currents corresponding to the nodes which are downstream to the desired node, as well as its own node; gives the current injected I(i) at that node.

8. After calculating the current injected to each bus, calculate the voltage of each bus using equation (12)

9. Compare the difference between each consecutive voltages values of every node. This gives deviation.

10. If deviation is less than or equal to tolerance limit, then update new voltage values and go to step 6. Else display absolute value of voltage and the phase angle.

11. Stop.

\subsection{Mathematical Modeling of DSTATCOM}

As noted earlier, in this paper, D-STATCOM is used for voltage regulation in the steady-state condition and can inject only reactive power to the system. Consequently, $I_{d s t a t}$ must be kept in quadrature with voltage of the system. By installing DSTATCOM in distribution system, all nodes voltage, especially the neighboring nodes of D-STATCOM location, and branches current of the network, change in the steady-state condition. The diagram of buses $i$ and $j$ of the distribution systems, when D-STATCOM is installed for voltage regulation in bus $j$, is shown in Fig.2. The phasor diagram of these buses with D-STATCOM effects is shown in Fig.3. Voltage of bus $j$ changes from $V_{j}$ to $V_{\text {jnew }}$ when D-STATCOM is used. For simplicity, the angle of voltage $V i$ is assumed to be zero. 


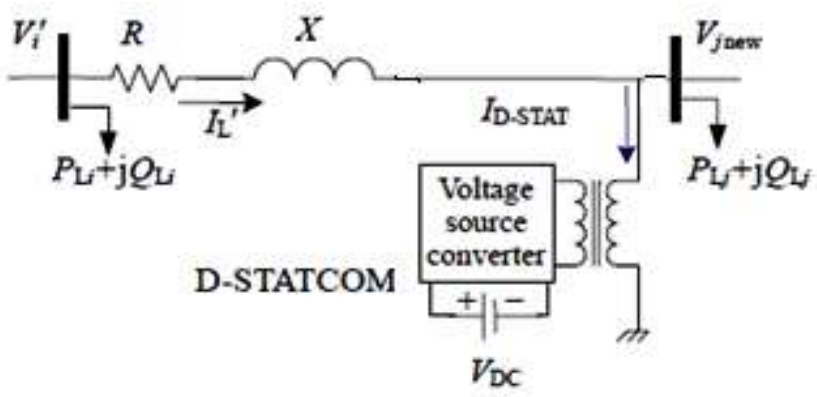

Figure 2: single line diagram of two buses with DSTATCOM

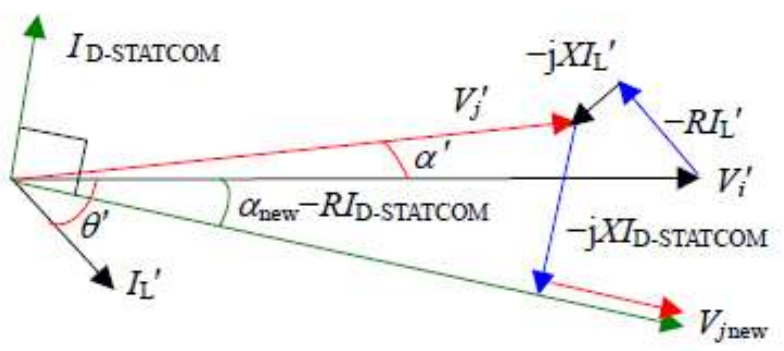

Figure 3 : phasor diagram voltages and currents

$$
V_{\text {jnew }} \angle \alpha_{\text {new }}=V_{i} \angle \delta-(R+j X) I_{L} \angle \theta-(R+j X) I_{\text {dstat }} \angle\left(\alpha_{\text {new }}+\frac{\pi}{2}\right)
$$

From the above equation (15) by equating imaginary and real parts we obtain

$$
\begin{aligned}
& V_{\text {jnew }} \cos \alpha_{\text {new }}=\operatorname{Re}\left(V_{i} \angle \delta\right)-\operatorname{Re}\left(R I_{L} \angle \theta\right)+X I_{\text {dstat }} \sin \left(\alpha_{\text {new }}+\frac{\pi}{2}\right)-R I_{\text {dstat }} \cos \left(\alpha_{\text {new }}+\frac{\pi}{2}\right) \\
& V_{\text {jnew }} \sin \alpha_{\text {new }}=\operatorname{Im}\left(V_{i} \angle \delta\right)-\operatorname{Im}\left(X I_{L} \angle \theta\right)-X I_{\text {dstat }} \cos \left(\alpha_{\text {new }}+\frac{\pi}{2}\right)-R I_{\text {dstat }} \sin \left(\alpha_{\text {new }}+\frac{\pi}{2}\right) \\
& a=\operatorname{Re}\left(V_{i} \angle \delta\right)-\operatorname{Re}\left(R I_{L} \angle \theta\right) \\
& b=\operatorname{Im}\left(V_{i} \angle \delta\right)-\operatorname{Im}(R X \angle \theta) \\
& C_{1}=-R, C_{2}=-X, d=V_{\text {jnew }} \\
& x_{1}=I_{\text {dstat }} \quad x_{2}=\alpha_{\text {new }}
\end{aligned}
$$

From equations (16), (17)

$$
\begin{aligned}
& d \cos x_{2}=a-c_{1} x_{1} \sin x_{1}-c_{2} x_{1} \cos x_{2} \\
& d \sin x_{2}=b-c_{2} x_{1} \sin x_{2}+c_{1} x_{1} \cos x_{2}
\end{aligned}
$$

$\mathrm{a}, \mathrm{b}, \mathrm{C}_{1}, \mathrm{C}_{2}$, are constants, we need to calculate $\mathrm{X}_{1}, \mathrm{X}_{2}$.

$$
x_{1}=\frac{d \cos x_{2}-a}{-c_{1} \sin x_{2}-c_{2} \cos x_{2}}, \quad x_{1}=\frac{d \sin x_{2}-b}{-c_{2} \sin x_{2}+c_{1} \cos x_{2}}
$$

Considering $\mathrm{x}=\sin \mathrm{X}_{2}$, Equating above equations (20) we get

$$
\left(k_{1}^{2}+k_{2}^{2}\right) x^{2}+\left(2 k_{1} d c_{1}\right) x+\left(d^{2} c_{1}^{2}-K_{2}^{2}\right)=0
$$

Where,

$k_{1}=a_{1} c_{2}-a_{2} c_{1}, \quad k_{2}=a_{1} c_{1}+a_{2} c_{2}$

The solution of equation (21) is given as,

$$
\begin{aligned}
& x=\left(-2 k_{1} d c_{1} \pm \sqrt{\left\{\left(2 k_{1} d c_{1}\right)^{2}-4\left(k_{1}^{2}+k_{2}^{2}\right)\left(d^{2} c_{1}^{2}-K_{2}^{2}\right)\right\}}\right. \\
& \alpha_{\text {new }}=x_{2}=\sin ^{-1} x
\end{aligned}
$$

Now the injected reactive power and voltage and current at node where is installed given as, 
$\overline{\text { jnew }}_{\text {jew }}=V_{\text {jnew }} \angle \alpha_{\text {new }}$

(23)

$$
\begin{aligned}
& I_{\text {dstat }}=I_{\text {dstat }} \angle\left(\alpha_{\text {new }}+\frac{\pi}{2}\right) \\
& j Q_{\text {dstat }}=V_{\text {jnew }} I_{\text {dstat }}^{8}
\end{aligned}
$$

and * denotes conjugate of complex variable.

The DSTATCOM is modeled such that the voltage magnitude node of the node where DSTATCOM is located is set to 1 p.u. The phase of the node where DSTATCOM is located is calculated by using equation (22), and the current flowing in DSTATCOM ie $\mathrm{I}_{\mathrm{dstat}}$ is calculated from equation (24). Finally the reactive power injected by DSTATCOM is calculated by equation (25).

Algorithm for radial distribution system load flow with DSTATCOM:

1. Read the distribution system line data and bus data.

2. Run the load flow of distribution system as in section 2.2

3. Select the candidate bus as in section 2.1

4. Assume the voltage profile of candidate bus as 1 p.u.

5. Calculate the injected reactive power of DSTATCOM and phase angle of the candidate bus using equations (22), (25).

6. Update the reactive power and voltage phase angle of compensated bus.

7. Run the load flow of distribution system with updated reactive power and phase angle of candidate bus.

8. end

\section{RESULTS AND DISCUSSION}

With the help of MATLAB program, the effectiveness of the proposed VSI and DSTATCOM performance is tested on $12.66 \mathrm{KV}, 100 \mathrm{MVA}$ radial distribution system consisting of 33 buses. The single line diagram of 33-bus system is shown in figure 4 and its data is given in [11].

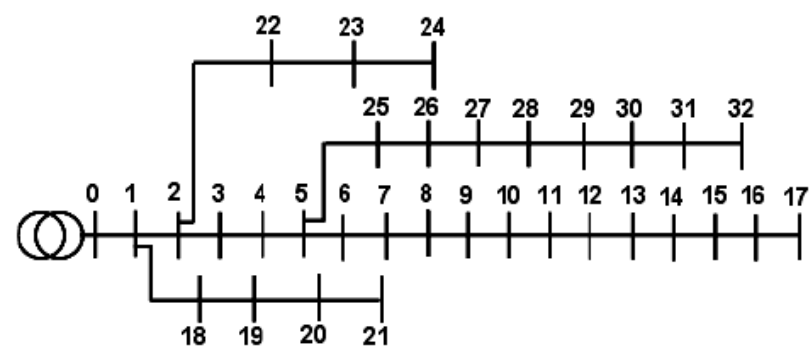

Figure 4: single line diagram of 33-bus distribution system.

The load flow of the 33 bus system is carried out and the voltages at each node and the Voltage Stability Indicator (VSI) are calculated and results shown in table below:

TABLE 1: Bus voltages and VSI values from load flow

\begin{tabular}{|c|c|c|c|}
\hline BUS & $\begin{array}{c}\text { Voltage (p.u.) } \\
\text { without } \\
\text { DSTATCOM }\end{array}$ & $\begin{array}{c}\text { Voltage } \\
\text { Stability } \\
\text { Indicator }\end{array}$ & $\begin{array}{c}\text { Voltage (p.u.) with } \\
\text { DSTATCOM at bus } \\
\text { 29 }\end{array}$ \\
\hline 0 & 1.0000 & - & 1.0000 \\
\hline 1 & 0.9970 & 0.0003 & 0.9983 \\
\hline 2 & 0.9830 & 0.0014 & 0.9909 \\
\hline 3 & 0.9755 & 0.0017 & 0.9885 \\
\hline 4 & 0.9682 & 0.0008 & 0.9863 \\
\hline 5 & 0.9498 & 0.0015 & 0.9861 \\
\hline 6 & 0.9463 & 0.0013 & 0.9831 \\
\hline 7 & 0.9415 & 0.0050 & 0.9784 \\
\hline 8 & 0.9352 & 0.0020 & 0.9726 \\
\hline 9 & 0.9294 & 0.0020 & 0.9671 \\
\hline
\end{tabular}


Identification of weak buses using Voltage Stability Indicator and its voltage profile improvement by

\begin{tabular}{|c|c|c|c|}
\hline 10 & 0.9286 & 0.0004 & 0.9663 \\
\hline 11 & 0.9271 & 0.0009 & 0.9648 \\
\hline 12 & 0.9210 & 0.0035 & 0.9591 \\
\hline 13 & 0.9187 & 0.0028 & 0.9571 \\
\hline 14 & 0.9173 & 0.0011 & 0.9558 \\
\hline 15 & 0.9160 & 0.0015 & 0.9546 \\
\hline 16 & 0.9140 & 0.0026 & 0.9528 \\
\hline 17 & 0.9134 & 0.0024 & 0.9522 \\
\hline 18 & 0.9965 & 0.0004 & 0.9978 \\
\hline 19 & 0.9929 & 0.0041 & 0.9942 \\
\hline 20 & 0.9922 & 0.0011 & 0.9935 \\
\hline 21 & 0.9916 & 0.0019 & 0.9929 \\
\hline 22 & 0.9794 & 0.0014 & 0.9874 \\
\hline 23 & 0.9727 & 0.0122 & 0.9808 \\
\hline 24 & 0.9694 & 0.0123 & 0.9775 \\
\hline 25 & 0.9479 & 0.0004 & 0.9870 \\
\hline 26 & 0.9453 & 0.0006 & 0.9884 \\
\hline 27 & 0.9339 & 0.0020 & 1.0005 \\
\hline 28 & 0.9257 & 0.0038 & 1.0002 \\
\hline 29 & 0.9222 & 0.0298 & 1.0010 \\
\hline 30 & 0.9180 & 0.0053 & 1.0005 \\
\hline 31 & 0.9171 & 0.0024 & 1.0097 \\
\hline 32 & 0.9168 & 0.0009 & 1.0095 \\
\hline
\end{tabular}

From the TABLE 1, it is noticed that the bus 29 has the highest value of VSI. Hence Bus 29 is considered to be the weak bus. And also bus 17 has the lowest voltage. The upper and lower limit of voltage magnitudes are 0.95 p.u. and 1.05 p.u. 21 out of 33 nodes have under voltage or over voltage problem when there is no DSATACOM installed.

In order to improve the voltage profile of the system the DSTATCOM is placed at the weak bus 29 . DSTATCOM is modeled such that it injects the required reactive power to maintain the voltage at 1 p.u. at the node where it is connected and improve voltage profile of other nodes. The load flow is carried out after placing DSTATCOM at node 29. TABLE 1 shows that after DSTATCOM improves the voltage of all the nodes having under voltage problems in distribution system. The result shows that the DSTATCOM installation in this node strongly improves the voltage of neighboring nodes.

TABLE 2: Active Power Loss of distribution system with and without DSTATCOM

\begin{tabular}{|c|c|c|}
\hline & Without & With DSTATCOM at \\
\hline Size of DSTATCOM (MVA) & - & 3.386 MVA \\
\hline No. buses with under or over & 21 & 0 \\
\hline Total Active Power losses (kw) & 201.8925 & 140.5936 \\
\hline \% loss reduction & - & $30.36 \%$ \\
\hline Cpu time (sec) & 0.083208 & 0.139495 \\
\hline
\end{tabular}

TABLE 2 shows the total Active power loss without DSTATCOM is $201.8925 \mathrm{KW}$, when DSTATCOM is placed at bus 29 the active power losses are reduced by $61.2929 \mathrm{KW}$. The active power loss reduction after DSTATCOM placement is 30.36\%. The results in TABLE 1 and 2 are achieved based on assumption that DSTATCOM has no capacity limit for reactive power injection to voltage compensation. And the size of DSTATCOM installed at bus 29 is 3.386 MVA. 


\section{CONCLUSION}

From the above simulation results, it is observed that node 29 has the highest value of VSI which is the weakest bus of 33 bus radial distribution system. Hence the DSTATCOM is placed at the weakest bus. After the DSTATCOM is placed the voltage profile of all the buses is improved. The DSATACOM is modeled such that it maintains voltage 1 p.u. at the bus where it is installed. Hence the voltage profile of the system is improved and system losses are minimized. As an extension of the work, the load multiplier factor may be increased and check the VSI performance. The DSTATCOM in this paper is modeled for fixed voltage and no capacity limit which may result in high MVA ratings.

\section{REFERENCES}

[1] H. K. Clark, "New challenges: Voltage stability" IEEE Power Engg Rev, April 1990, pp. 33-37.

[2] T. Van Cutsem: "A method to compute reactive power margins with respect to voltage collapse", IEEE Trans. On Power Systems, No. 1, 1991

[3] Ph.D thesis of Dr. C.K. Chanda on "Global voltage stability indicator index" in 2003,BESU.

[4] T.K.Abdul, G.B.Jasmon "A New Technique for Voltage Stability Analysis in a Power system \& Improved Load Flow Algorithm for Distribution Network”. IEEE Catalogue no 95TH8130

[5] Chanda, S.; Das, B.; , "Identification of weak buses in a power network using novel voltage stability indicator in radial distribution system," International Conference on Power Electronics (IICPE), 2010 India, vol., no., pp.1-4, 28-30 Jan. 2011 doi: 10.1109/IICPE.2011.5728121

[6] C.K. Chanda, A. Chakraborti,S.Dey, "Development of global voltage security indicator(VSI) and role of SVC on it in longitudinal power supply(LPS) system”, ELSEVIER(Electrical Power System Research 68),2004, pp.1 -9.

[7] Bishop, M.T., Foster, J.D., Down, D.A., 1994. The Application of Single-phase Voltage Regulators on Three-phase Distribution Systems. The 38th Annual Conf. on Rural Electric Power, p.C2/1-C2/7.

[8] Gu, Z., Rizy, D.T., 1996. Neural networks for combined control of capacitor banks and voltage regulators in distribution systems. IEEE Trans. on Power Delivery, 11:1921- 1928. [doi:10.1109/61.544277].

[9] Kojovic, L.A., 2006. Coordination of Distributed Generation and Step Voltage Regulator Operations for Improved Distribution System Voltage Regulation. IEEE Power Engineering Society General Meeting, p.232-237.

[10] Ramsay, S.M., Cronin, P.E., Nelson, R.J., Bian, J., Menendez, F.E., 1996. Using Distribution Static Compensators (DSTATCOMs) to Extend the Capability of Voltage-limited Distribution Feeders. The 39th Annual Conf. on Rural Electcic power, p.18-24

[11] Haque, M.H., 2001. Compensation of Distribution System Voltage Sag by DVR and D-STATCOM. IEEE Porto Power Tech. Conf., 1(5):223-228.

[12] Ghosh, S., Das, D., 1999. Method for load-flow solution of radial distribution networks. IEE Proc.-Gener. Transm. Distrib., 146(6):641-648. [doi:10.1049/ip-gtd:19990464]

[13] Ma, J., Xu, J., Wang, S., Lin, X., 2002. Calculation and Analysis for Line Losses in Distribution Network. Int. Conf. on Power System Technology, p.2537-2541.

[14] Baran, M.E., Wu, F.F., 1989b. Network reconfiguration in distribution systems for loss reduction and load balancing. IEEE Trans. on Power Delivery, 4(2):1401-1407 